Network Working Group

Request for Comments: 4856

S. Casner

Obsoletes: 3555

Packet Design

Category: Standards Track

March 2007

\title{
Media Type Registration of Payload Formats in the RTP Profile for Audio and Video Conferences
}

Status of This Memo

This document specifies an Internet standards track protocol for the Internet community, and requests discussion and suggestions for improvements. Please refer to the current edition of the "Internet Official Protocol standards" (STD 1) for the standardization state and status of this protocol. Distribution of this memo is unlimited.

Copyright Notice

Copyright (C) The IETF Trust (2007).

Abstract

This document specifies media type registrations for the RTP payload formats defined in the RTP Profile for Audio and Video Conferences. Some of these may also be used for transfer modes other than RTP.

Table of Contents

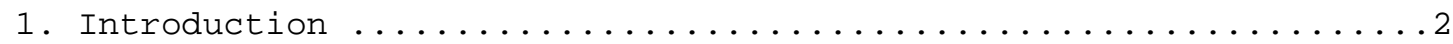

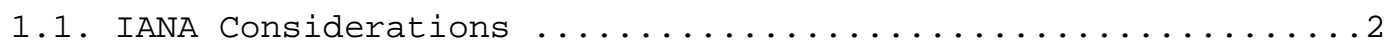

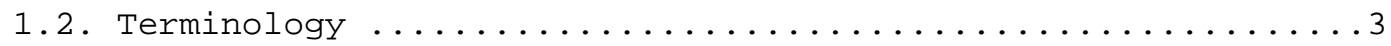

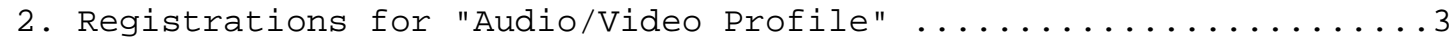

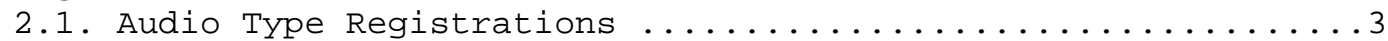

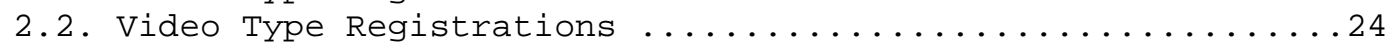

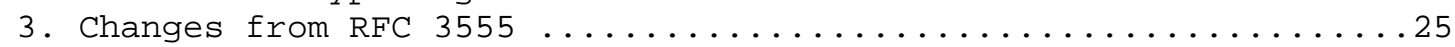

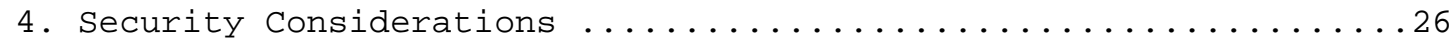

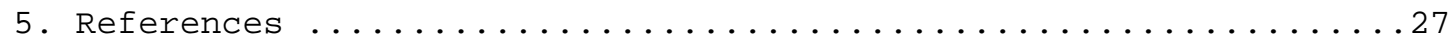

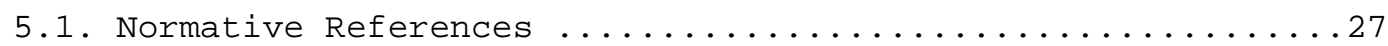

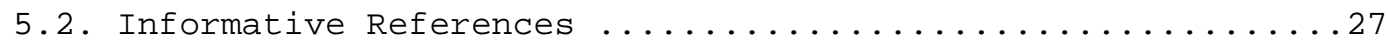




\section{Introduction}

This document updates the media type registrations initially specified in RFC 3555 for the Real-time Transport Protocol (RTP) payload formats defined in the RTP Profile for Audio and Video Conferences, RFC 3551 [1], as subtypes under the "audio" and "video" media types. This document does not include media type registrations for the RTP payload formats that are referenced in RFC 3551 but defined in other RFCs. The media type registrations for those payload formats are intended to be updated by including them in revisions of the individual RFCs defining the payload formats.

The media type registrations specified here conform to the updated template format and procedures in RFC 4288 [2] and RFC 4855 [3]. This update makes no technical changes in the registrations. Together with RFC 4855, this document obsoletes RFC 3555.

\subsection{IANA Considerations}

As a consequence of the generalized applicability of the media types registry as specified in RFC 4288, some changes in nomenclature are needed in the RTP Payload Format section of the registry. In the registry title "RTP Payload Format MIME types" and the introductory text, "MIME" should be changed to "media". "MIME" should be deleted from the table headings, leaving just "media type" and "subtype".

This document updates the media type registrations listed below to conform to the revised registration format specified in $\mathrm{RFC} 4288$ and RFC 4855, so the reference for these media types should be changed from RFC 3555 to this document. Some media type registrations contained in RFC 3555 are omitted from this document; the existing registrations for those types continue to be valid until updated by other RFCs. There are no new registrations contained here. 


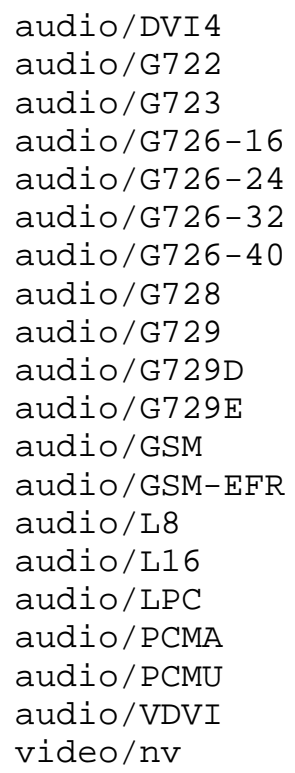

\subsection{Terminology}

The key words "MUST", "MUST NOT", "REQUIRED", "SHALL", "SHALL NOT", "SHOULD", "SHOULD NOT", "RECOMMENDED", "MAY", and "OPTIONAL" in this document are to be interpreted as described in RFC 2119 [4] and indicate requirement levels for implementations compliant with this specification.

\section{Registrations for "Audio/Video Profile"}

In the following sections, the RTP payload formats defined in the RTP Profile for Audio and Video Conferences, RFC 3551 [1], are registered as media types.

\subsection{Audio Type Registrations}

For most audio payload formats, the RTP timestamp clock rate is equal to the sampling rate. Some payload formats operate only at one fixed sampling rate, while others are adjustable.

These audio formats also include the optional parameters "ptime" to specify the recommended length of time in milliseconds represented by 
the media in a packet, and "maxptime" to specify the maximum amount of media that can be encapsulated in each packet, expressed as time in milliseconds. The "ptime" and "maxptime" parameters are defined in the Session Description Protocol (SDP), RFC 4566 [5].

\subsubsection{Registration of Media Type audio/DVI4}

Type name: audio

Subtype name: DVI4

Required parameters: rate: The RTP timestamp clock rate, which is equal to the sampling rate. The typical rate is 8000 , but other rates may be specified.

Optional parameters: ptime, maxptime (see RFC 4566)

Encoding considerations: This media type is framed binary data (see section 4.8 in RFC $4288)$.

Security considerations: This media type does not carry active content. It does transfer compressed data. See Section 4 of RFC 4856.

Interoperability considerations: none

Published specification: RFC 3551

Applications that use this media type: Audio and video streaming and conferencing tools.

Additional information: none

Person \& email address to contact for further information: Stephen Casner <casnerdacm.org>

Intended usage: COMMON

Restrictions on usage:

This media type depends on RTP framing, and hence is only defined for transfer via RTP (RFC 3550 [6]). Transfer within other framing protocols is not defined at this time.

Author:

Stephen Casner 


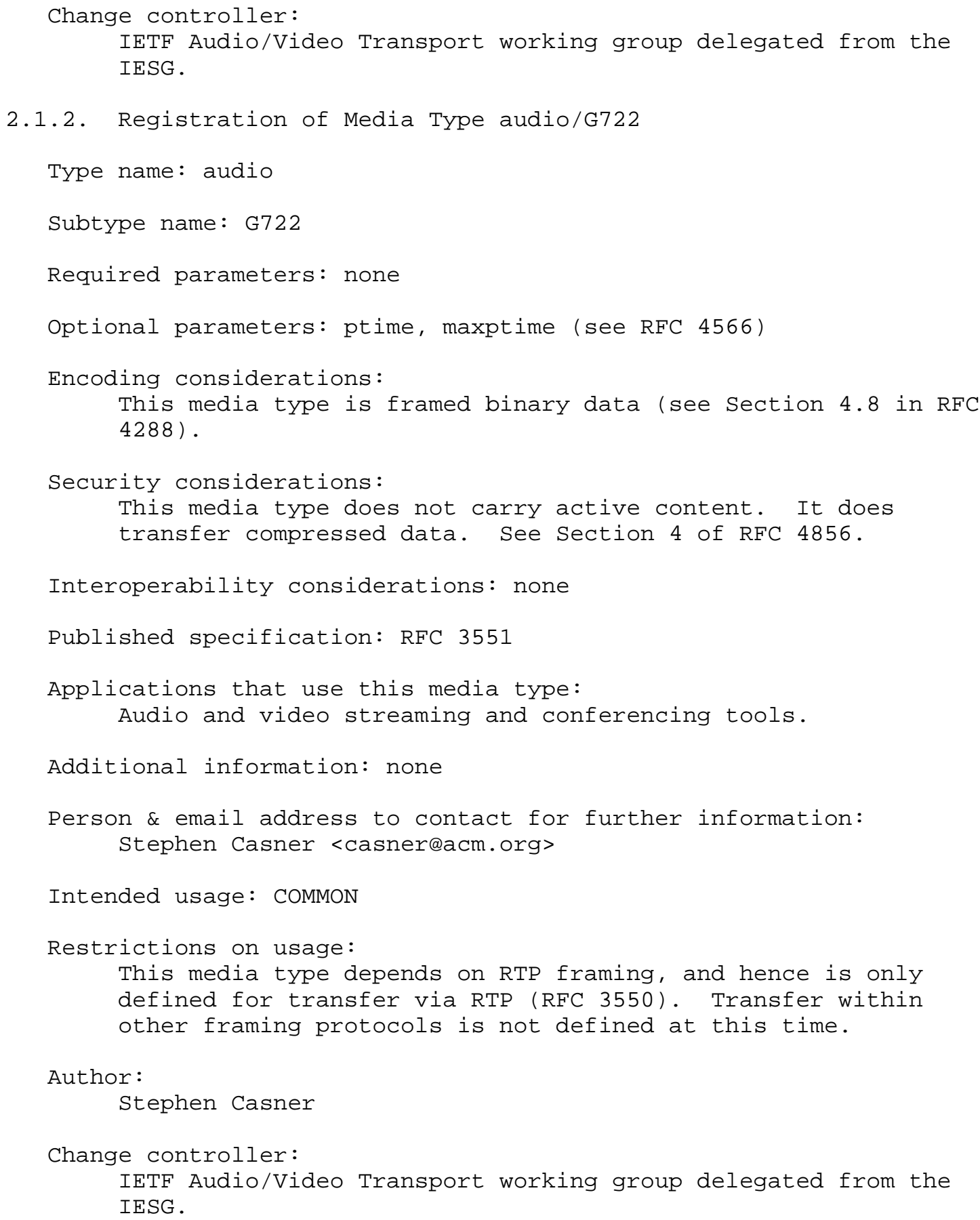




\subsubsection{Registration of Media Type audio/G723}

Type name: audio

Subtype name: G723

Required parameters: none

Optional parameters:

ptime, maxptime: see RFC 4566

bitrate: the data rate in $\mathrm{kb} / \mathrm{s}$ used or preferred for the audio

bit stream, with permissible values 5.3 or 6.3 . If

unspecified, the bitrate may change from frame to frame as

indicated inband.

annexa: indicates that Annex A, voice activity detection, is

used or preferred. Permissible values are "yes" and "no" (without the quotes); "yes" is implied if this parameter is omitted.

Encoding considerations:

This media type is framed binary data (see section 4.8 in RFC $4288)$.

Security considerations:

This media type does not carry active content. It does

transfer compressed data. See Section 4 of RFC 4856.

Interoperability considerations: none

Published specification: RFC 3551

Applications that use this media type:

Audio and video streaming and conferencing tools.

Additional information: none

Person \& email address to contact for further information:

Stephen Casner <casnereacm.org>

Intended usage: COMMON

Restrictions on usage:

This media type depends on RTP framing, and hence is only defined for transfer via RTP (RFC 3550). Transfer within other framing protocols is not defined at this time. 
Author:

Stephen Casner

Change controller:

IETF Audio/Video Transport working group delegated from the IESG.

2.1.4. Registration of Media Type audio/G726-16

Type name: audio

Subtype name: G726-16

Required parameters: none

Optional parameters: ptime, maxptime (see RFC 4566)

Encoding considerations:

This media type is framed binary data (see section 4.8 in RFC $4288)$.

Security considerations:

This media type does not carry active content. It does

transfer compressed data. See Section 4 of RFC 4856.

Interoperability considerations: none

Published specification: RFC 3551

Applications that use this media type:

Audio and video streaming and conferencing tools.

Additional information: none

Person \& email address to contact for further information:

Stephen Casner <casnereacm.org>

Intended usage: COMMON

Restrictions on usage:

This media type depends on RTP framing, and hence is only defined for transfer via RTP (RFC 3550). Transfer within other framing protocols is not defined at this time.

Author:

Stephen Casner 


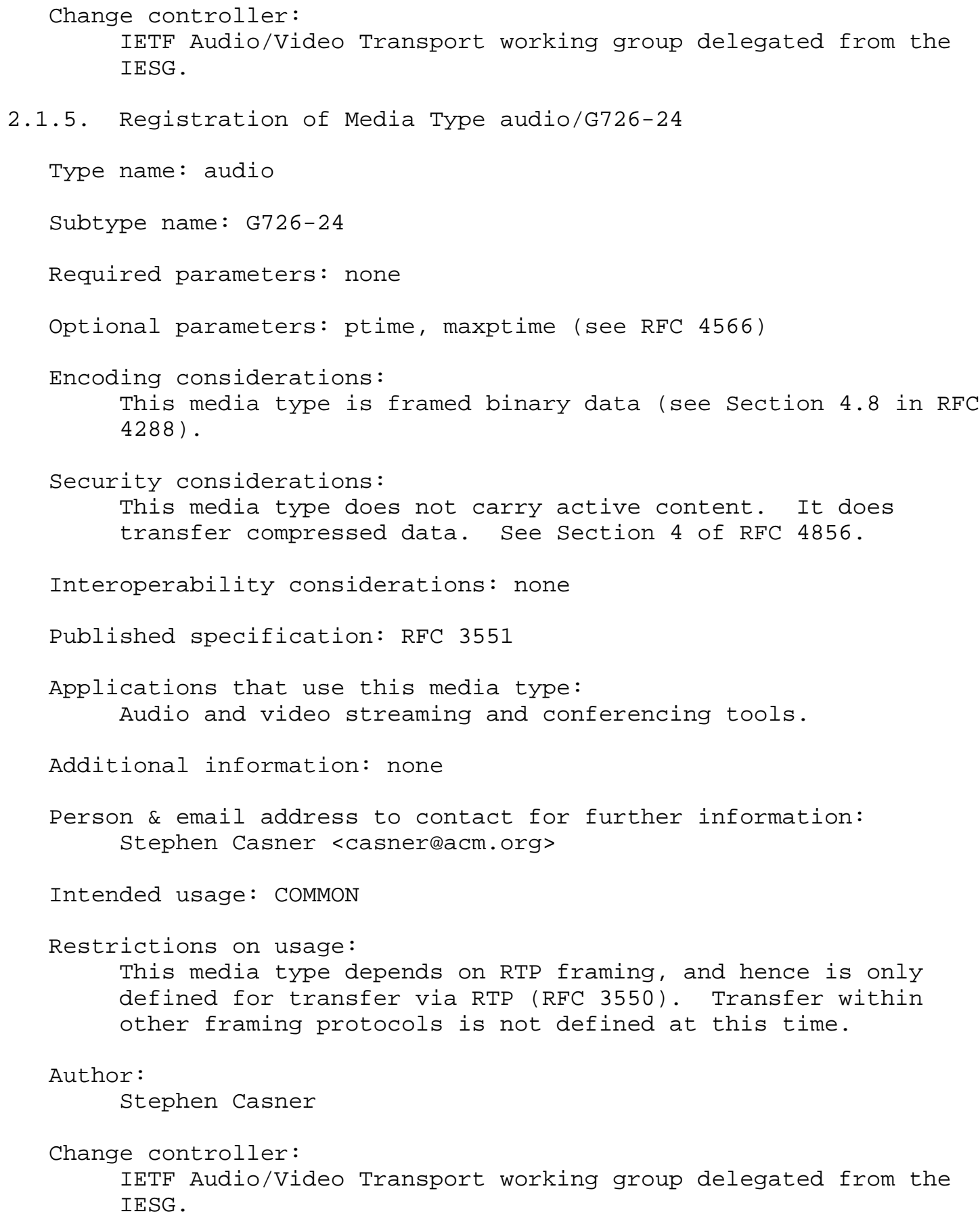




\subsubsection{Registration of Media Type audio/G726-32}

Type name: audio

Subtype name: G726-32

Required parameters: none

Optional parameters: ptime, maxptime (see RFC 4566)

Encoding considerations:

This media type is framed binary data (see section 4.8 in RFC $4288)$.

Security considerations:

This media type does not carry active content. It does

transfer compressed data. See Section 4 of RFC 4856.

Interoperability considerations: none

Published specification: RFC 3551

Applications that use this media type: Audio and video streaming and conferencing tools.

Additional information: none

Person \& email address to contact for further information: Stephen Casner <casnerlacm.org>

Intended usage: COMMON

Restrictions on usage:

This media type depends on RTP framing, and hence is only defined for transfer via RTP (RFC 3550). Transfer within other framing protocols is not defined at this time.

Author:

Stephen Casner

Change controller: IETF Audio/Video Transport working group delegated from the IESG. 


\subsubsection{Registration of Media Type audio/G726-40}

Type name: audio

Subtype name: G726-40

Required parameters: none

Optional parameters: ptime, maxptime (see RFC 4566)

Encoding considerations:

This media type is framed binary data (see section 4.8 in RFC $4288)$.

Security considerations:

This media type does not carry active content. It does

transfer compressed data. See Section 4 of RFC 4856.

Interoperability considerations: none

Published specification: RFC 3551

Applications that use this media type: Audio and video streaming and conferencing tools.

Additional information: none

Person \& email address to contact for further information: Stephen Casner <casnerlacm.org>

Intended usage: COMMON

Restrictions on usage:

This media type depends on RTP framing, and hence is only defined for transfer via RTP (RFC 3550). Transfer within other framing protocols is not defined at this time.

Author:

Stephen Casner

Change controller: IETF Audio/Video Transport working group delegated from the IESG. 


\subsubsection{Registration of Media Type audio/G728}

Type name: audio

Subtype name: G728

Required parameters: none

Optional parameters: ptime, maxptime (see RFC 4566)

Encoding considerations:

This media type is framed binary data (see section 4.8 in RFC $4288)$.

Security considerations:

This media type does not carry active content. It does

transfer compressed data. See Section 4 of RFC 4856.

Interoperability considerations: none

Published specification: RFC 3551

Applications that use this media type: Audio and video streaming and conferencing tools.

Additional information: none

Person \& email address to contact for further information: Stephen Casner <casnerlacm.org>

Intended usage: COMMON

Restrictions on usage:

This media type depends on RTP framing, and hence is only defined for transfer via RTP (RFC 3550). Transfer within other framing protocols is not defined at this time.

Author:

Stephen Casner

Change controller: IETF Audio/Video Transport working group delegated from the IESG. 


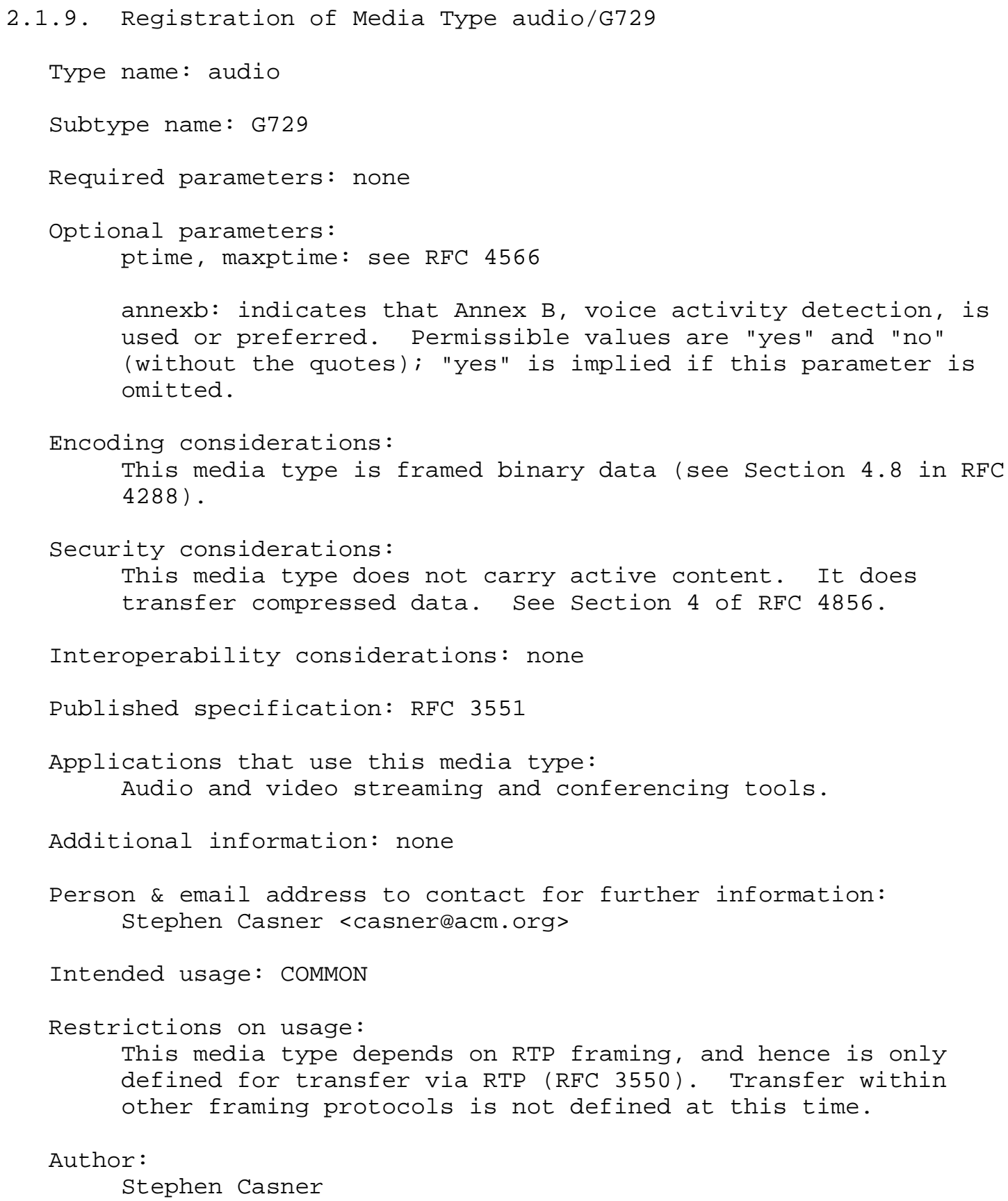




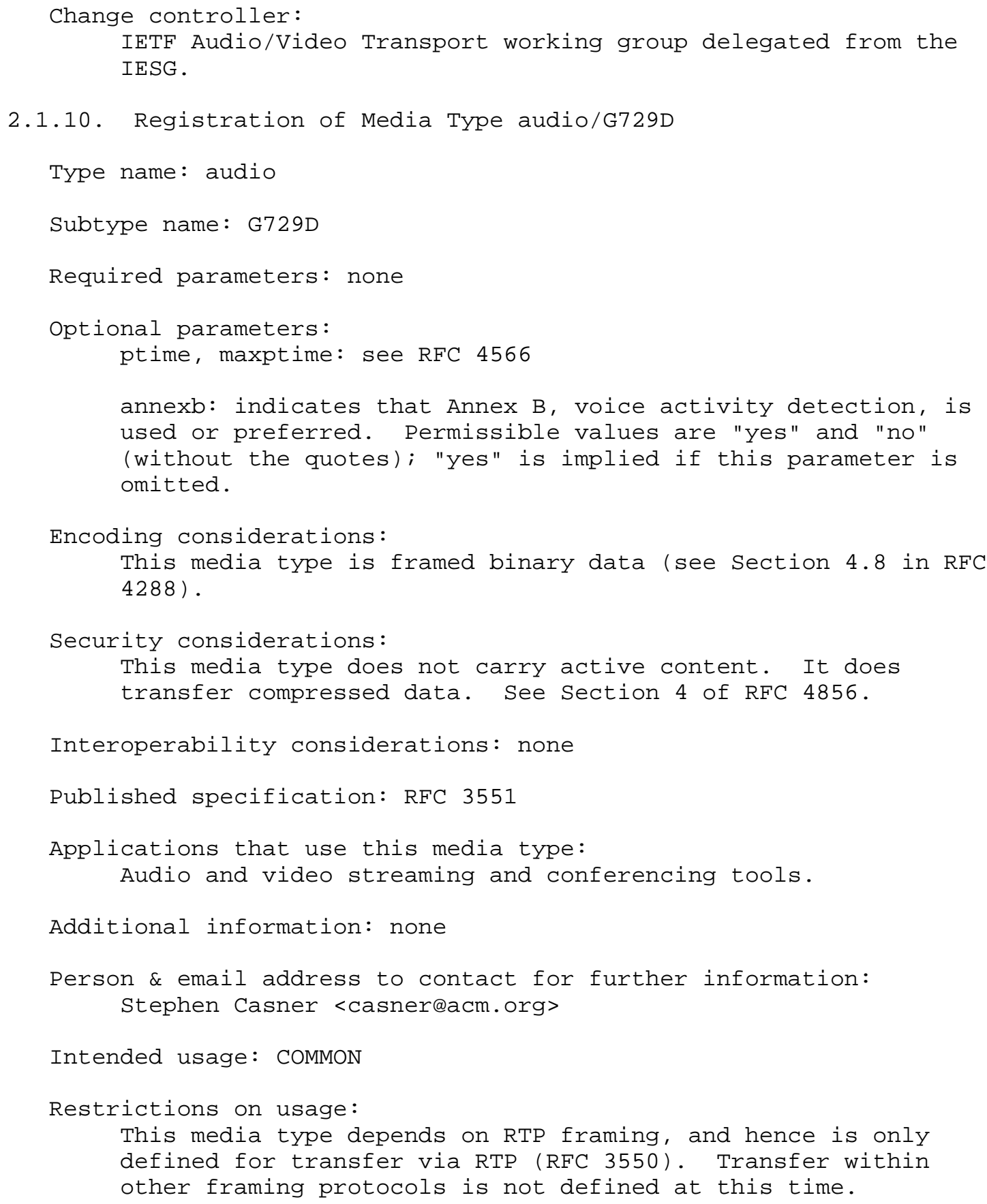


Author:

Stephen Casner

Change controller:

IETF Audio/Video Transport working group delegated from the IESG.

2.1.11. Registration of Media Type audio/G729E

Type name: audio

Subtype name: G729E

Required parameters: none

Optional parameters:

ptime, maxptime: see RFC 4566

annexb: indicates that Annex B, voice activity detection, is used or preferred. Permissible values are "yes" and "no" (without the quotes); "yes" is implied if this parameter is omitted.

Encoding considerations:

This media type is framed binary data (see section 4.8 in RFC $4288)$.

Security considerations:

This media type does not carry active content. It does transfer compressed data. See Section 4 of RFC 4856.

Interoperability considerations: none

Published specification: RFC 3551

Applications that use this media type: Audio and video streaming and conferencing tools.

Additional information: none

Person \& email address to contact for further information: Stephen Casner <casnerlacm.org>

Intended usage: COMMON 


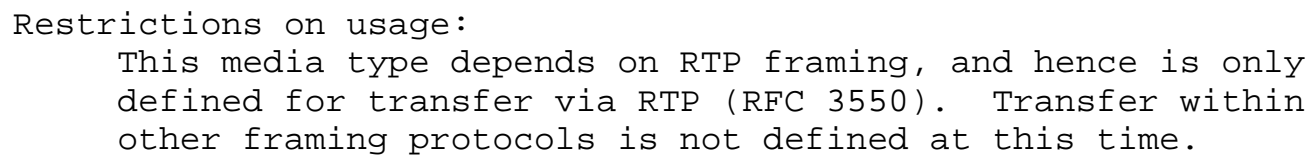


Author:

Stephen Casner

Change controller:

IETF Audio/Video Transport working group delegated from the IESG.

2.1.13. Registration of Media Type audio/GSM-EFR

Type name: audio

Subtype name: GSM-EFR

Required parameters: none

Optional parameters: ptime, maxptime (see RFC 4566)

Encoding considerations:

This media type is framed binary data (see section 4.8 in RFC $4288)$.

Security considerations:

This media type does not carry active content. It does

transfer compressed data. See Section 4 of RFC 4856.

Interoperability considerations: none

Published specification: RFC 3551

Applications that use this media type:

Audio and video streaming and conferencing tools.

Additional information: none

Person \& email address to contact for further information:

Stephen Casner <casnereacm.org>

Intended usage: COMMON

Restrictions on usage:

This media type depends on RTP framing, and hence is only defined for transfer via RTP (RFC 3550). Transfer within other framing protocols is not defined at this time.

Author:

Stephen Casner 


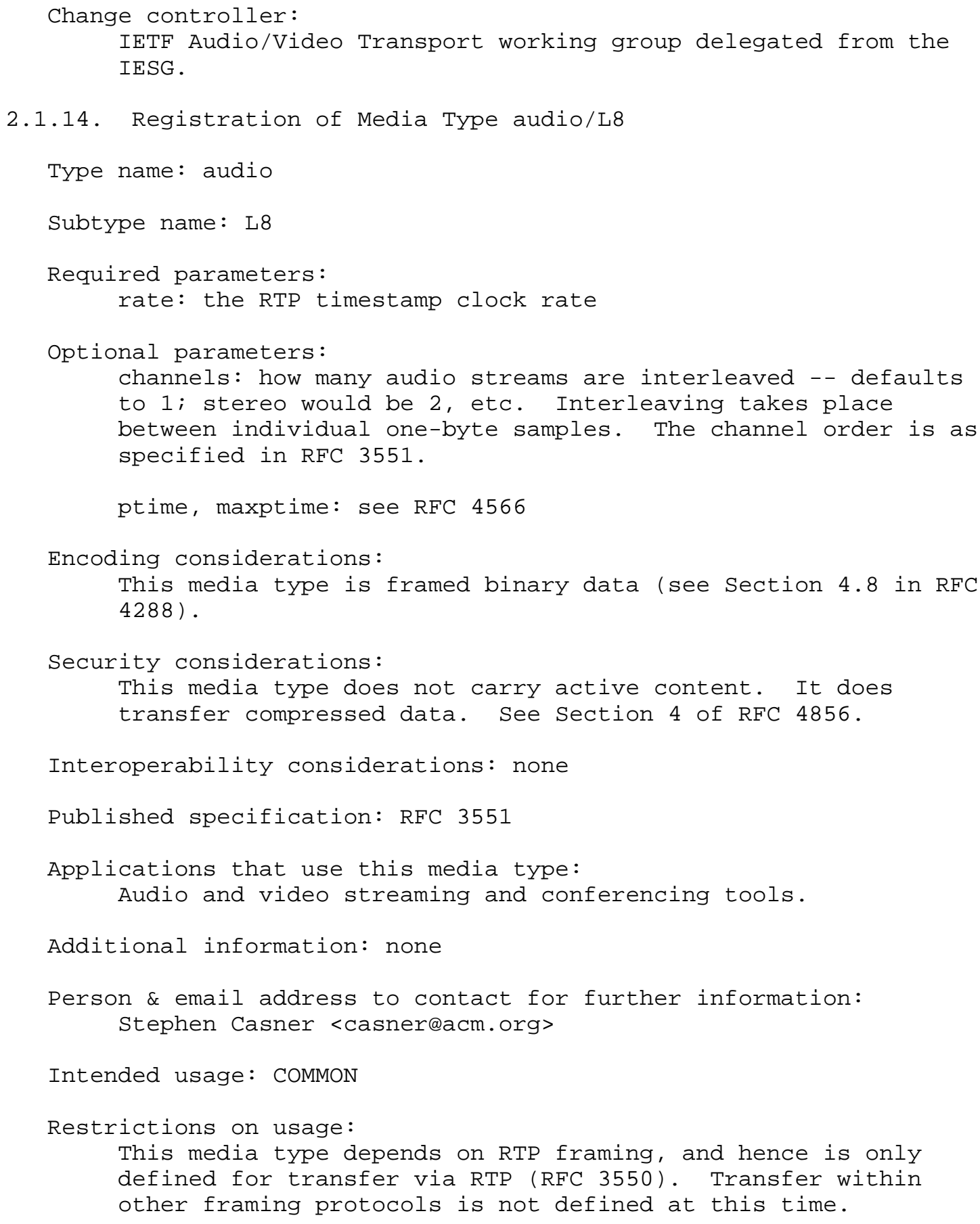


Author:

Stephen Casner

Change controller:

IETF Audio/Video Transport working group delegated from the IESG.

\subsubsection{Registration of Media Type audio/L16}

Media type audio/L16 was initially registered via RFC 2586 [10] for transports other than RTP. That registration is incorporated here and augmented with additional information for RTP transport.

Type name: audio

Subtype name: L16

Required parameters:

rate: number of samples per second -- For non-RTP transport, the permissible values for rate are $8000,11025,16000,22050$, $24000,32000,44100$, and 48000 samples per second. For RTP transport, other values are permissible but the aforementioned values are RECOMMENDED. For RTP, the rate parameter indicates the RTP timestamp clock rate, which is equal to the sample rate.

Optional parameters:

channels: how many audio streams are interleaved -- defaults to 1 ; stereo would be 2 , etc. Interleaving takes place between individual two-byte samples. The channel order is as specified in RFC 3551 unless a channel-order parameter is also present.

emphasis: analog preemphasis applied to the signal before quantization. The only emphasis value defined here is emphasis $=50-15$ to indicate the 50/15 microsecond preemphasis used with Compact Discs. This parameter MUST be omitted if no analog preemphasis was applied. Note that this is a stream property parameter, not a receiver configuration parameter. Thus, if parameters are negotiated, it may not be possible for the sender to comply with a receiver request for a particular setting.

channel-order: specifies the sample interleaving order for multiple-channel audio streams (see RFC 3190 [7], Section 7). Permissible values are DV.LRLsRs, DV.LRCS, DV.LRCWo, DV.LRLsRsC, DV.LRLsRsCS, DV.LmixRmixTWOQ1Q2, DV.LRCWoLsRsLmixRmix, DV.LRCWoLs1Rs1Ls2Rs2, DV.LRCWoLsRsLcRc. 
For interoperation with DV video systems, only a subset of these channel combinations is specified for use with 20-bit linear encoding in the DV video specification [9]; those are DV.LRLsRs, DV.LRCS, DV.LmixRmixTWoQ1Q2. This parameter MUST be omitted when the AIFF-C channel order convention (see RFC 3551) is in use.

For RTP, ptime: RECOMMENDED duration of each packet in milliseconds.

For RTP, maxptime: maximum duration of each packet in milliseconds.

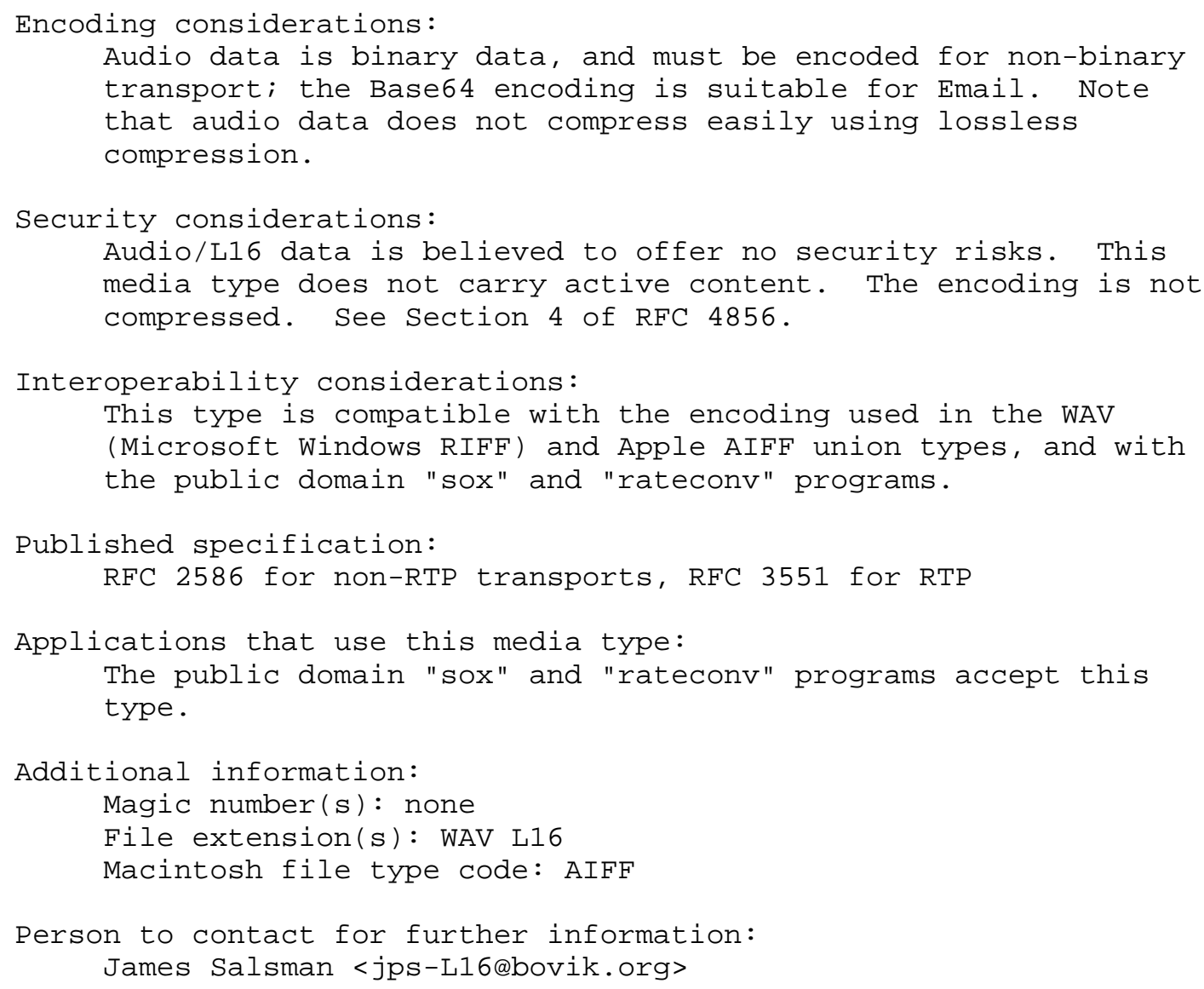


Intended usage:

Common

It is expected that many audio and speech applications will

use this type. Already the most popular platforms provide

this type with the rate=11025 parameter, referred to as "radio quality speech".

Restrictions on usage:

In addition to file-based transfer methods, this type is also defined for transfer via RTP (RFC 3550).

Author:

James Salsman for non-RTP transports.

Stephen Casner for RTP transport.

Change controller:

James Salsman for non-RTP transports.

For RTP transport, IETF Audio/Video Transport working group

delegated from the IESG.

2.1.16. Registration of Media Type audio/LPC

Type name: audio

Subtype name: LPC

Required parameters: none

Optional parameters: ptime, maxptime (see RFC 4566)

Encoding considerations:

This media type is framed binary data (see section 4.8 in RFC $4288)$.

Security considerations:

This media type does not carry active content. It does

transfer compressed data. See Section 4 of RFC 4856.

Interoperability considerations: none

Published specification: RFC 3551

Applications that use this media type:

Audio and video streaming and conferencing tools.

Additional information: none 


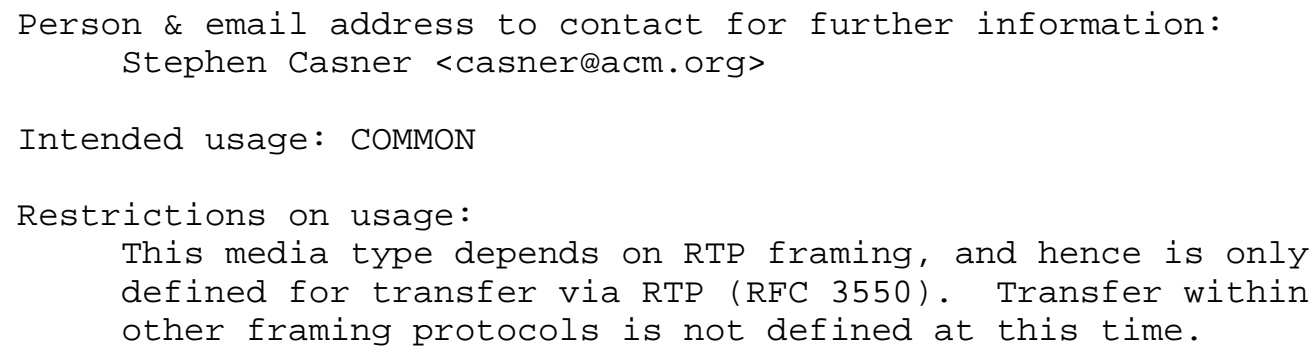




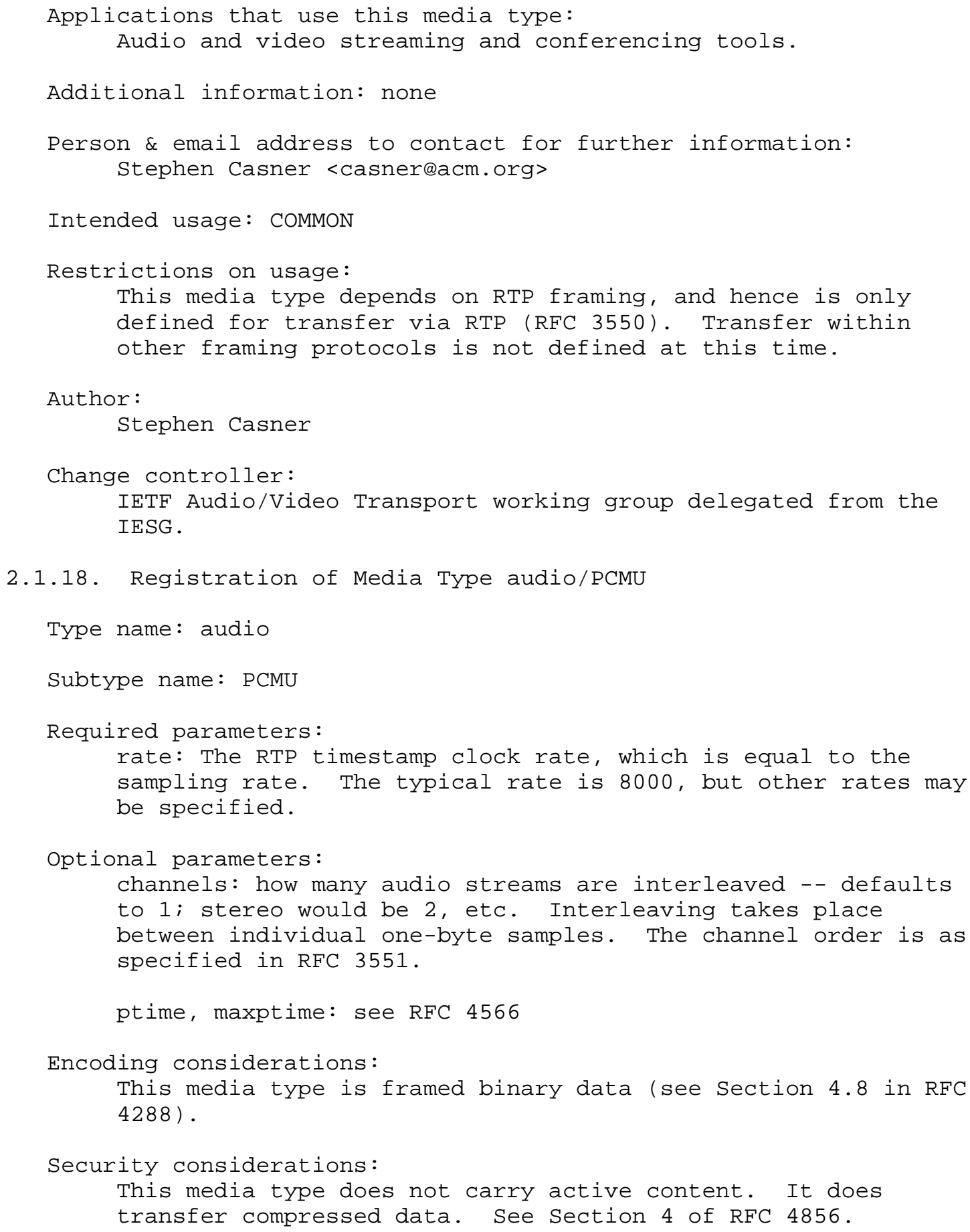


Interoperability considerations: none

Published specification: RFC 3551

Applications that use this media type:

Audio and video streaming and conferencing tools.

Additional information: none

Person \& email address to contact for further information:

Stephen Casner <casnereacm.org>

Intended usage: COMMON

Restrictions on usage:

This media type depends on RTP framing, and hence is only defined for transfer via RTP (RFC 3550). Transfer within other framing protocols is not defined at this time.

Author:

Stephen Casner

Change controller:

IETF Audio/Video Transport working group delegated from the IESG.

2.1.19. Registration of Media Type audio/VDVI

Type name: audio

Subtype name: VDVI

Required parameters: none

Optional parameters: ptime, maxptime (see RFC 4566)

Encoding considerations:

This media type is framed binary data (see section 4.8 in RFC $4288)$.

Security considerations:

This media type does not carry active content. It does transfer compressed data. See Section 4 of RFC 4856.

Interoperability considerations: none

Published specification: RFC 3551 


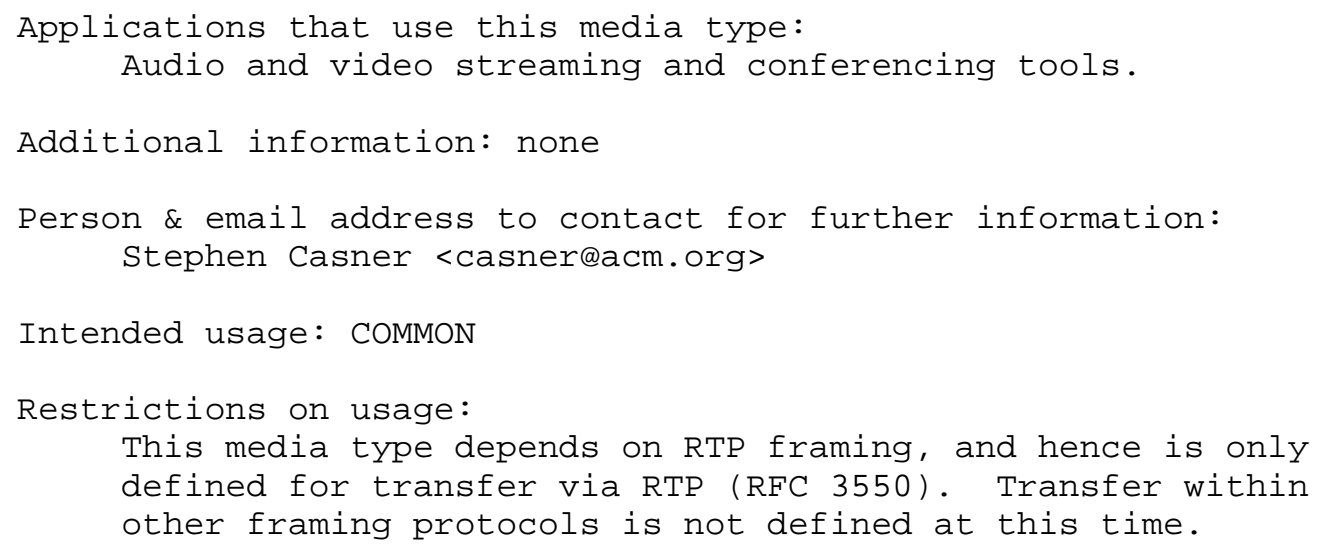

2.2. Video Type Registrations

For most video payload formats, including the one registered here, the RTP timestamp clock rate is always $90000 \mathrm{~Hz}$, so the "rate" parameter is not applicable. Likewise, the "channel" parameter is not used with video, while "ptime" and "maxptime" could be but typically are not.

2.2.1. Registration of Media Type video/nv

Type name: video

Subtype name: nv

Required parameters: none

Optional parameters: none

Encoding considerations: This media type is framed binary data (see Section 4.8 in RFC 4288 ).

Security considerations:

This media type does not carry active content. It does transfer compressed data. See Section 4 of RFC 4856. 
Interoperability considerations: none

Published specification: RFC 3551

Applications that use this media type:

Audio and video streaming and conferencing tools.

Additional information: none

Person \& email address to contact for further information:

Stephen Casner <casnereacm.org>

Intended usage: COMMON

Restrictions on usage:

This media type depends on RTP framing, and hence is only defined for transfer via RTP (RFC 3550). Transfer within other framing protocols is not defined at this time.

Author:

Stephen Casner

Change controller:

IETF Audio/Video Transport working group delegated from the IESG.

3. Changes from RFC 3555

RFC 3555 is obsoleted by the combination of RFC 4855 [3] and this document. RFC 4855 retains the specification of procedures and requirements from RFC 3555, while the media type registrations from RFC 3555 were extracted into this document. The media type registrations for the RTP payload formats that are referenced in $\mathrm{RFC}$ 3551 [1], but defined in other RFCs, have been elided from this document because those registrations are intended to be updated by including them in revisions of the individual RFCs defining the payload formats.

The media type registrations in this document have been updated to conform to the revised media type registration procedures in $\mathrm{RFC} 4288$ [2] and RFC 4855. Whereas RFC 3555 required the encoding considerations to specify transfer via RTP, that is now specified under restrictions on usage. The encoding considerations now warn that these types are framed binary data. The change controller is also now identified according to current conventions. The optional parameter "channels" was clarified for audio subtypes L8, PCMA, and PCMU. Finally, reference [9], which was missing from RFC 3555, has been corrected. 


\section{Security Considerations}

This memo specifies media type registrations for the transfer of several compressed audio and video data encodings via RTP, so implementations using these media types are subject to the security considerations discussed in the RTP specification [8].

None of these media types carry "active content" that could impose malicious side-effects upon the receiver. The content consists solely of compressed audio or video data to be decoded and presented as sound or images. However, several audio and video encodings are perfect for hiding data using steganography.

A potential denial-of-service threat exists for data encodings using compression techniques that have non-uniform receiver-end computational load. The attacker can inject pathological datagrams into the stream, which are complex to decode and cause the receiver to be overloaded. However, none of the encodings registered here has an expansion factor greater than about 20, and all are considered relatively simple by modern standards (some are implemented on handheld devices and most were implemented on general-purpose computers ten years ago).

As with any IP-based protocol, in some circumstances a receiver may be overloaded simply by the receipt of too many packets, either desired or undesired. Network-layer authentication MAY be used to discard packets from undesired sources, but the processing cost of the authentication itself may be too high.

RTP may be sent via IP multicast, which provides no direct means for a sender to know all the receivers of the data sent and therefore no measure of privacy. Rightly or not, users may be more sensitive to privacy concerns with audio and video communication than they have been with more traditional forms of network communication. Therefore, the use of security mechanisms with RTP to provide confidentiality and integrity of the data is important. Because the data compression used with these media types is applied end-to-end, encryption may be performed after compression so there is no conflict between the two operations. 


\section{References}

\subsection{Normative References}

[1] Schulzrinne, H. and S. Casner, "RTP Profile for Audio and Video Conferences with Minimal Control", RFC 3551, July 2003.

[2] Freed, N. and J. Klensin, "Media Type Specifications and Registration Procedures", BCP 13, RFC 4288, December 2005.

[3] Casner, S., "Media Type Registration of RTP Payload Types", RFC 4855, January 2007 .

[4] Bradner, S., "Key words for use in RFCs to Indicate Requirement Levels", BCP 14, RFC 2119, March 1997.

[5] Handley, M., Jacobson, V., and C. Perkins, "SDP: Session Description Protocol", RFC 4566, July 2006.

[6] Schulzrinne, H., Casner, S., Frederick, R. and V. Jacobson, "RTP: A Transport Protocol for Real-Time Applications", RFC 3550, July 2003 .

[7] Kobayashi, K., Ogawa, A., Casner, S. and C. Bormann, "RTP Payload Format for 12-bit DAT Audio and 20- and 24-bit Linear Sampled Audio", RFC 3190, January 2002.

[8] Schulzrinne, H., Casner, S., Frederick, R. and V. Jacobson, "RTP: A Transport Protocol for Real-Time Applications", RFC 3550, July 2003 .

\subsection{Informative References}

[9] IEC 61834, Helical-scan digital video cassette recording system using 6,35 mm magnetic tape for consumer use (525-60, 625-50, 1125-60, and 1250-50 systems), August 1998 .

[10] Salsman, J. and H. Alvestrand, "The Audio/L16 MIME content type", RFC 2586, May 1999. 


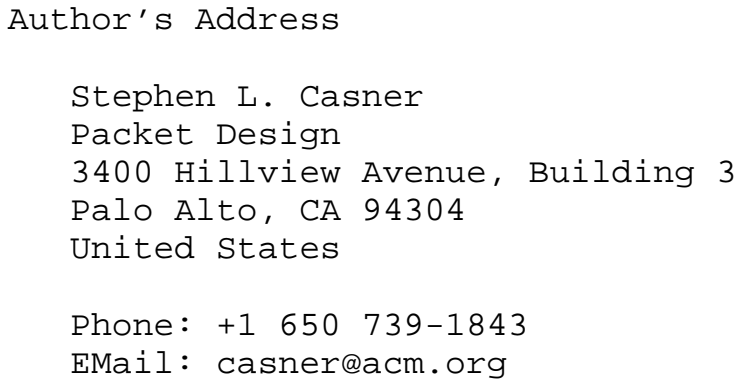


Full Copyright statement

Copyright (C) The IETF Trust (2007).

This document is subject to the rights, licenses and restrictions contained in BCP 78, and except as set forth therein, the authors retain all their rights.

This document and the information contained herein are provided on an "AS IS" basis and THE CONTRIBUTOR, THE ORGANIZATION HE/SHE REPRESENTS OR IS SPONSORED BY (IF ANY), THE INTERNET SOCIETY, THE IETF TRUST AND THE INTERNET ENGINEERING TASK FORCE DISCLAIM ALL WARRANTIES, EXPRESS OR IMPLIED, INCLUDING BUT NOT LIMITED TO ANY WARRANTY THAT THE USE OF THE INFORMATION HEREIN WILL NOT INFRINGE ANY RIGHTS OR ANY IMPLIED WARRANTIES OF MERCHANTABILITY OR FITNESS FOR A PARTICULAR PURPOSE.

Intellectual Property

The IETF takes no position regarding the validity or scope of any Intellectual Property Rights or other rights that might be claimed to pertain to the implementation or use of the technology described in this document or the extent to which any license under such rights might or might not be available; nor does it represent that it has made any independent effort to identify any such rights. Information on the procedures with respect to rights in RFC documents can be found in BCP 78 and BCP 79 .

Copies of IPR disclosures made to the IETF Secretariat and any assurances of licenses to be made available, or the result of an attempt made to obtain a general license or permission for the use of such proprietary rights by implementers or users of this specification can be obtained from the IETF on-line IPR repository at http://www.ietf.org/ipr.

The IETF invites any interested party to bring to its attention any copyrights, patents or patent applications, or other proprietary rights that may cover technology that may be required to implement this standard. Please address the information to the IETF at ietf-ipreietf.org.

Acknowledgement

Funding for the RFC Editor function is currently provided by the Internet Society. 